\title{
Des hommes malades du chômage ? Genre et (ré- )assignation identitaire au Royaume-Uni
}

Men sick with unemployment? Gender and identity (re)assignation in the

United-Kingdom

\section{Tania Angeloff}

\section{OpenEdition}

\section{Journals}

Édition électronique

URL : http://journals.openedition.org/travailemploi/5476

DOI : 10.4000/travailemploi.5476

ISSN : 1775-416X

Éditeur

DARES - Ministère du Travail

Édition imprimée

Date de publication : 15 novembre 2011

Pagination : 69-82

ISBN : 0224-4365

ISSN : 0224-4365

\section{Référence électronique}

Tania Angeloff, « Des hommes malades du chômage ? Genre et (ré-)assignation identitaire au

Royaume-Uni », Travail et Emploi [En ligne], 128 | octobre-décembre 2011, mis en ligne le 12 septembre 2012, consulté le 01 mai 2019. URL : http://journals.openedition.org/travailemploi/5476 ; DOI :

10.4000/travailemploi.5476 


\title{
Des hommes malades du chômage? Genre et (ré-)assignation identitaire au Royaume-Uni
}

\author{
Tania Angeloff(*)
}

\begin{abstract}
En Angleterre, au début des années 2000, près de deux millions d'hommes touchent une prestation d'adulte handicapé (contre moins d'un million de femmes). Relevant du dispositif d'Incapacity Benefit, promu au début des années 1980, ils sont administrativement et principalement perçus comme des adultes handicapés, jusqu'à ce que soit lancé, en 2001, un programme de retour à l'emploi, intitulé New Deal For Disabled People. A l'intérieur de l'Agence nationale d'emploi (Jobcentre Plus), des centres de recherche d'emploi (Job Broking Service) leur sont spécifiquement dédiés. À partir d'une enquête réalisée en 2004 dans le nord de l'Angleterre, l'article interroge les questions de réassignation identitaire de ces hommes "malades», très souvent à la suite de ruptures d'emploi, en s'intéressant aux interactions entre des bénéficiaires de l'Incapacity Benefit, incités à reprendre un emploi, et des conseillers emplois censés les aider dans cette démarche. Comment une injonction administrative joue-t-elle dans la constitution ou l'aménagement d'une identité sociale? Les conseillers, en invitant les allocataires à penser à un travail « dans l'idéal» tout en étant "réalistes», édictent l'injonction paradoxale propre au dispositif qui débouche souvent sur des emplois médiocres, voire bénévoles. Les réactions des participants à cette structure révèlent des négociations identitaires variables selon le genre, l'âge et le profil professionnel, mais aussi selon le degré de handicap.
\end{abstract}

La prestation d'adulte handicapé (Incapacity Benefit) est, au Royaume-Uni, un phénomène sexué qui, jusqu'à une date très récente, a concerné une forte majorité d'hommes. Le nord de l'Angleterre, lourdement affecté par la crise économique et la restructuration des années 1980, a ainsi connu une inactivité massive au masculin, en liaison avec l'invalidité - ici comprise à la fois comme catégorie administrative et comme situation sociale. En 2001, une politique d'emploi voit le jour (New Deal for Disabled People), destinée à remettre au travail une grande partie des inactifs percevant l'Incapacity Benefit. À partir d'une enquête de terrain réalisée en 2004 dans un «centre de réinsertion par l'emploi» (Job broking service), je me suis intéressée au phénomène de (re-)construction identitaire du point de vue des bénéficiaires ${ }^{(1)}$ de l'Incapacity Benefit. Les questions ayant motivé cette étude ne portaient pas directement sur les mécanismes institutionnels de reconversion sociale et professionnelle des allocataires, même s'il me paraît incontournable de les préciser ici. La recherche a davantage été guidée par une problématique identitaire, en termes d'effets d'assignation, de rupture et d'appropriation. Sans les citer toutes, différentes approches sociologiques

(*) Université Paris-Dauphine, IRISSO; Tania.Angeloff@ dauphine.fr

(1) Les termes «bénéficiaire» et «allocataire» s'entendront ici au sens strict pour renvoyer aux individus bénéficiant de l'Incapacity Benefit que les travailleurs sociaux et les conseillers emploi appellent, quant à eux, des «clients». se sont déjà intéressées au «halo du chômage» (FreYsSinet, 2004) et à la précarité laborieuse, sous divers angles, dans le contexte social français. Ces études sont éclairantes pour comprendre, d'une part, la construction sociale des catégories d'emploi, de chômage et d'inactivité (Salais, Baverez, 1986; Maruani, 2002; Demazière, 2003; Paugam, 2007); d'autre part, les négociations identitaires concernant les chômeurs et bénéficiaires de dispositifs d'insertion dans l'emploi (SCHNAPPER, 1981; Demazière, 1992; Roinsart, 2007 ; Duvoux, 2009).

Dans la continuité de ces diverses approches, je souhaitais comprendre comment, dans un pays considéré comme un marché du travail «libéral», les dispositifs institutionnels sont mobilisés, instrumentalisés ou rejetés par des personnes en invalidité sommées de se remettre au travail. En d'autres termes, en quoi le statut institutionnel d'un-e bénéficiaire de l'Incapacity Benefit poussé(e) à (ré) intégrer le marché du travail affecte-t-il sa construction identitaire? Comment les hommes rencontrés se définissent-ils avant tout : inactifs ou demandeurs d'emploi, ou encore les deux suivant les contextes? Et pourquoi ces dispositifs concernent-ils en grande majorité des hommes? À quels stéréotypes de genre, de classe et d'âge ce processus renvoie-t-il ?

Dans une première partie, j'exposerai le contexte dans lequel le dispositif de l'Incapacity Benefit a vu le jour, en rappelant les principales évolutions du marché du travail au Royaume-Uni en matière de chômage ces trente dernières années. Dans ce 
cadre, je dégagerai le processus de catégorisation inhérent au dispositif d'Incapacity Benefit en soulignant ses ambiguiités. Dans une seconde partie, en m'appuyant plus particulièrement sur l'enquête de terrain réalisée, je m'intéresserai aux effets du travail de catégorisation administrative et sociale sur les principaux concernés - les hommes inactifs et aux interactions avec leurs conseillers emploi, en m'attachant à dégager des éléments de la négociation identitaire.

\section{Le dispositif de l'Incapacity Benefit et ses enjeux dans le contexte britannique}

\section{Chômage et "emploi convenable" sur le marché du travail britannique (2)}

L'Incapacity Benefit est né dans un contexte de montée du chômage en Grande-Bretagne et reste étroitement lié à la problématique de l'emploi et de l'inactivité. Ce n'est pas un hasard si le dispositif a vu le jour au Royaume-Uni au début des années 1980, en lien avec les progrès technologiques, quand le système de recension des chômeurs s'est informatisé. À partir de cette date, seuls sont comptabilisés comme chômeurs ceux qui perçoivent des allocations chômage. Ce nouveau mode de comptage national (différent du comptage du Bureau international du travail( $\left.{ }^{(3)}\right)$ permet ainsi de réduire le taux de chômage, pour la seule année 1982, de 3,7 \% (Denman, McDonald, 1996 cité par Alcock et al., 2003, p. 7). En outre, ce classement (juridique, politique et statistique) exclut ceux et celles qui, n'ayant jamais travaillé, souhaiteraient le faire mais ne perçoivent aucune indemnité. Cela explique un taux de chômage particulièrement bas au Royaume-Uni, de l'ordre de $5 \%$ depuis 2001 (BegG, LeFresne, 2008).

Le chômage frappe diversement en fonction de la région, du sexe, de l'âge, de l'origine ethnique et de la reconnaissance éventuelle d'un handicap. Cela est notamment lié à la structure de l'emploi, différente d'un comté à l'autre. De plus, au tournant des années 1980, sous le gouvernement Thatcher, est initiée une vague de privatisations, parallèlement à un processus de désindustrialisation déjà amorcé quelques années auparavant. Dès lors, la

(2) Il ne s'agit pas ici d'entrer dans le détail de l'évolution du marché du travail en matière de chômage, d'inactivité, de préretraite, etc., mais uniquement de rappeler quelques grandes lignes permettant de situer et de comprendre les enjeux du dispositif de l'Incapacity Benefit.

(3) Selon la définition du BIT, est chômeur toute personne en âge de travailler (quinze ans ou plus) sans emploi durant la semaine de référence, disponible pour en prendre un dans les quinze jours et déclarant en avoir cherché un activement dans le mois précédent ou en avoir trouvé un qui commence dans moins de trois mois. structure de l'emploi se modifie et conduit à un taux de chômage masculin supérieur au taux de chômage des femmes. Entre 1979 et 1998, d'après l'Office for National Statistics, 2,7 millions d'emplois masculins auraient disparu. Dans le même temps, les emplois occupés par les femmes ont crû de 2,2 millions entre 1981 et 1998 , bien que cette croissance ait dissimulé un fort taux de temps partiel(4) et masqué les licenciements massifs de femmes dans l'industrie. Le nord de l'Angleterre, fortement industriel, a donc été touché de plein fouet par cette évolution structurelle.

Dans les années 1990, le département des Affaires sociales a connu des restructurations importantes qui voient fusionner, à la fin de la décennie, les services d'enregistrement et de suivi des chômeurs avec les services de versement de l'allocation chômage dans une nouvelle structure appelée Jobcentre Plus (BEGG, LeFresne, 2008). Dans le même mouvement, en 1996, l'«allocation chômage»a été remplacée par une allocation de «demandeur d'emploi», dans une logique où la reconnaissance collective du droit à l'emploi se déplace vers une responsabilité individuelle du chômage (LeFresNe, 2008). Le changement de terminologie, symboliquement important, montre à quel point l'idéologie de l'État-providence (welfare) s'effrite au profit de l'encouragement d'une éthique du travail (workfare), comme c'est le cas un peu partout en Europe (Demazière, 2003; Duvoux, Paugam, 2008).

Il en résulte une redéfinition de l'« emploi convenable» dont l'acception devient plus large, et qui intègre des emplois précaires en expansion, qualifiés outre-Manche de bad jobs (emplois pourris), de dead-end jobs (emplois impasses), autrement dit de "miettes d'emplois», avec une flexibilité du temps de travail et des salaires et une faible protection sociale (5) (Angeloff, 1999; Lindsay, McQuaid, 2004; BegG, Lefresne, 2008). L'activation du nouveau dispositif d'emploi, au nom d'une injonction à travailler à tout prix, justifie l'orientation des chômeurs vers des emplois à temps partiel courts, très mal rémunérés, voire vers des emplois bénévoles dans le secteur non marchand qui sont institutionnellement valorisés.

Cette politique lancée par l'administration de Tony Blair, dès son arrivée au pouvoir en 1997, a fait débat. Elle a contribué à ériger le RoyaumeUni en modèle pour son faible taux de chômage, quand la réalité se traduisait principalement par une augmentation du taux d'inactivité liée, d'une part,

(4) Seules $57 \%$ des femmes actives occupées britanniques travaillent alors à temps complet (EUROSTAT, 1983-2000).

(5) Jusqu'en 1999, en Angleterre, il n'existait pas de salaire minimum et nombre de travailleurs étaient des «workers» sans protection sociale, car ils n'avaient pas atteint un niveau minimal de salaire ou d'heures de travail pour être reconnus comme des «employees». 
au retrait des femmes de l'emploi, d'autre part, à un important basculement des hommes au chômage vers le statut d'invalide ou d'adulte handicapé.

\section{Des hommes en retrait du marché du travail}

Dans ce contexte, on a en effet observé un phénomène de «retrait» du marché du travail (detachment) d'un nombre grandissant d'hommes. Par «retrait» ou « détachement», les sociologues britanniques du travail entendent la disparition du marché du travail d'hommes qui devraient y être, y travailler ou y chercher un emploi, et s'en sont comme absentés (YeAndle, in Alcock et al., 2003).

Les régions les plus touchées sont le pays de Galles, le comté de Merseyside (qui a Liverpool pour capitale), la région de Manchester, le sud du Yorkshire, le nord-est de l'Angleterre et Clydeside, encore plus au nord. Dans ces régions, la part des bénéficiaires de l'aide sociale excède $20 \%$ de la population masculine en âge de travailler alors que dans de nombreuses régions du sud de l'Angleterre ce chiffre se situe en dessous des $5 \%$. En outre, ce sont également les régions les plus touchées par le chômage qui sont le plus affectées par le dispositif d'invalidité (Alcock, Beatty et al., 2003). Ce phénomène renvoie, d'une part, aux liens avérés entre chômage et maladie (BARNAY, 2008; KHLAT, Sermet, 2011), d'autre part, aux «trappes d'inactivité», expression désignant une situation où l'individu inactif n'a financièrement pas intérêt à accepter un emploi qui serait au mieux aussi bien rémunéré que son allocation chômage ou ses minima sociaux (Benarrosh, 2003; Dubet, Vérétout, 2001). Compte tenu du faible montant des prestations sociales, ces trappes d'inactivité constituent aussi des «trappes à pauvreté» (Duvoux, 2009).

Depuis trois décennies, le retrait du marché du travail des hommes - estimé plus important que celui des femmes - constitue sans conteste l'un des changements sociaux les plus marquants au Royaume-Uni. Parmi ces absents de l'emploi, dans les années 1990, il existe environ deux millions d'hommes relevant de l'assurance maladie ${ }^{(6)}$. En d'autres termes, parmi les individus percevant des prestations pour invalidité, souvent cumulées à d'autres allocations sociales ${ }^{(7)}$, on trouve un grand nombre d'hommes «employables», qui veulent travailler ou retravailler ou sont fortement incités à le faire et ont accès aux offres d'emploi des agences nationales s'ils le souhaitent, tout en étant statistiquement catégorisés comme «inactifs».
Ce phénomène de retrait apparaît d'autant plus préoccupant qu'il contribue à minimiser le chômage réel. Parallèlement aux conséquences des atteintes à la santé sur le travail, avec des cas de maladie subie ou masquée (Gollac, VolkofF, 2006) pour ceux qui sont dans l'emploi, on rencontre un chômage masqué par la maladie, ou plus exactement occulté par les catégories de «maladie», d' «invalidité» ou de «handicap». Au Royaume-Uni, l'assurance maladie et le dispositif de préretraite ont servi de réponses à la hausse du chômage ces trente dernières années.

\section{L'Incapacity Benefit : une réponse à la crise de l'emploi?}

Né au début des années 1980, le dispositif de l'Incapacity Benefit - «allocation maladie» ou «allocation d'invalidité» - est complexe. Produit d'une histoire qui remonte au XIX ${ }^{\mathrm{e}}$ siècle avec la loi contre la pauvreté (Poor Law Amendment Act) de 1834 , le dispositif en porte la marque encore aujourd'hui et reste régi par le principe de moindre éligibilité. Les allocations doivent rester faibles afin que la personne assistée et sans emploi perçoive moins d'argent que le moins bien payé des actifs occupés ${ }^{(8)}$.

La complexité de 1'Incapacity Benefit tient également au chevauchement entre les prestations sociales et aides sociales : allocation maladie, doublée parfois d'indemnisation du chômage ou d'un revenu d'insertion; allocations familiales, allocation logement, exonération des impôts locaux ${ }^{(9)}$.

En outre, la composition des allocataires n'est pas homogène du point de vue du handicap. En 2003-2004, sur les 2,6 millions de bénéficiaires sans emploi et en âge de travailler, certains ont un handicap lourd les empêchant de travailler $(10 \%)$, d'autres n'ont pas de réel handicap physique ou mental mais sont en situation de très grande pauvreté $(30 \%)$ et la majorité regroupe ceux dont le handicap est considéré comme léger ou moyen et qui peuvent retravailler sous certaines conditions (60\%) (BEATTY et al., 2009). À noter que dans ce système, les accidents du travail relèvent d'un régime particulier, indépendant de l'Incapacity Benefit.

Enfin, du point de vue du genre, le dispositif a toujours touché plus d'hommes que de femmes même si, ces toutes dernières années, elles tendent à rattraper les hommes (BEATTY et al., 2009).
(6) Le chiffre diminue au tournant des années 2000. Pour indication, en 2003-2004, 1,3 million d'hommes ont demandé l'Incapacity Benefit, contre 800000 femmes (source BEATTY, FotHERGILL, Houston, 2009).

(7) Tels l'allocation logement, le revenu minimum d'insertion (alloué sous condition de ressources), ou l'exonération de la taxe foncière et d'habitation.
(8) Il faut attendre 1999 au Royaume-Uni pour que soit fixé un salaire minimum horaire.

(9) Dans les années 1990, moins de 20 \% des chômeurs comptaient uniquement sur l'allocation chômage et plus de $60 \%$ dépendaient d'une prestation complémentaire en raison de leurs faibles revenus (Alcock, BEATty et al., 2003). 
Si au départ, le mécanisme de l'Incapacity Benefit semble avoir été conçu pour répondre à une situation où le chômage et la précarité au travail augmentaient, conjointement à des problèmes de santé perçus comme persistants (dépressions, troubles musculosquelettiques), il a ensuite été victime de son succès et s'est fortement développé. Des études ont montré qu'il existait une corrélation entre chômage et santé (Barnay, 2008; Khlat, Sermet, 2011), et que cette dernière était plus précaire - subjectivement et objectivement - dans les régions les plus touchées par le chômage (Bellaby, 1999). Cette corrélation suggère un cercle vicieux : une santé précaire fragilise un retour dans l'emploi tandis que l'absence durable d'emploi renforce la maladie, ce que confirment les entretiens que j'ai menés.

\section{Sortir du cercle vicieux : la mise en place de programmes New Deals}

Dans un mouvement commun à l'ensemble des pays occidentaux depuis vingt-cinq ans, l'accent est mis sur les facteurs individuels de la pauvreté et sur la nécessité d'《activer» des politiques sociales pour réformer un système de protection perçu comme trop généreux par les gouvernants (DAVOINE, 2005; Lefresne, 2008; Duvoux, 2009). Au début des années 2000, le gouvernement de Tony Blair lance une série de programmes dans le cadre des différents types de Jobcentre Plus, programmes qualifiés de New Deals et déclinés différemment suivant les situations : ainsi, pour les jeunes de seize à vingt-quatre ans, un dispositif est mis en place, le New Deal for Young People, pour les inciter à gagner le marché du travail. De même, les plus de cinquante-cinq ans sont la cible d'une politique incitative pour retrouver un emploi, le New Deal Plus. Les New Deals semblent fleurir au gré des catégories : New Deal for Lone Parents, New Deal for Musicians, New Deal for Partners. Toutes les populations ciblées sont encouragées à se rendre au centre d'emploi le plus proche de chez elles pour rencontrer un-e conseiller-e emploi ${ }^{(10)}$ censé-e les aider à réintégrer le marché du travail. Au sein de ces dispositifs, certains représentent une obligation, d'autres reposent sur le volontariat.

Une telle évolution traduit une pratique générale en Europe, en lien avec les transformations de l'emploi, l'extension du "précariat», selon l'expression de Robert CASTEL, et les mutations de la protection sociale. Ces transformations impliquent la promotion d'un " "sujet actif" que [les] services de conseil et d'orientation et d'accompagnement outillent» (Divay, Perez, 2012, p. 61.) L'allocataire est encouragé à devenir l'acteur de sa réinsertion (EBERSOLD,

(10) Le terme «conseiller emploi» est directement traduit ici de job adviser, titre donné à la fois aux travailleurs sociaux des centres $J O B S$ (voir encadré 1) et aux conseillers privés des agences de placement qui viennent ponctuellement.
2004) au nom d'une éthique de la responsabilité (Duvoux, 2009). En 2001, le programme New Deal for Disabled People voit ainsi le jour, parallèlement à la structure des Job Broking Services. Leurs personnels ont vocation à accompagner les personnes répertoriées handicapées ou en maladie, et à mettre en œuvre le programme de recherche d'emploi dans des structures spécifiquement dédiées aux personnes en invalidité, plus petites que les centres d'emploi classiques, et reposant sur le volontariat des allocataires, sans sanction pour ceux ayant plus de deux ans d'ancienneté dans le dispositif, soit l'écrasante majorité des allocataires.

Notons que le programme New Deal for Disabled People a connu un succès très mitigé puisque, selon un rapport de 2005, entre le lancement du dispositif à l'échelle nationale, en juillet 2001, et mars 2005 (soit au moment de l'enquête, voir encadré 1), seuls 146340 allocataires se sont inscrits (KAZIMIRSKI et al., 2005) sur un total de 2,7 millions d'allocataires. Pour la majorité des inscrits, il s'agissait de bénéficiaires de sexe masculin, prêts à retravailler, jeunes, percevant l'allocation depuis moins de deux ans, davantage sujets à des troubles musculo-squelettiques qu'à des problèmes mentaux. Sur la même période, $39 \%$ d'entre eux avaient trouvé un emploi rémunéré, dont $70 \%$ trois mois après s'être inscrits.

\section{Encadré 1 \\ Une enquête localisée dans le nord de l'Angleterre}

Les résultats présentés ici sont ceux d'une enquête réalisée dans le nord de l'Angleterre durant l'année 2004. Elle a été menée dans une petite ville située à une vingtaine de kilomètres de Manchester qui fait administrativement partie de ce que les Anglais appellent le «Greater Manchester».

L'objectif de l'enquête était, d'une part, de comprendre le dispositif New Deal for Disabled People mis en place trois ans auparavant; d'autre part, d'étudier l'interface entre des inactifs relevant d'un dispositif social de prise en charge d'invalidité - permanente ou temporaire - appelé Incapacity Benefit et les conseillers d'un centre de réinsertion dans et par l'emploi (Job Broking Service ${ }^{(11)}$ ou JOBS) censés les aider à retrouver une activité professionnelle rémunérée ou bénévole. Mon questionnement visait à articuler les logiques institutionnelles, incarnées par les conseillers emploi, et les stratégies identitaires des allocataires, socialement inactifs et en longue maladie.

Le centre enquêté se composait d'une gestionnaire, d'une psychologue, d'une secrétaire et de cinq conseillers emploi dépendant de l'agence nationale pour l'emploi. À l'occasion, des conseillers issus d'agences privées y venaient également et

(11) « To broke », c'est jouer le tout pour le tout en anglais. Dans ce contexte, le JOBS se présente comme l'antenne emploi de la dernière chance, celle où l'on n'a rien à perdre et où l'on a tout à gagner. 
participaient à certaines réunions. Enfin, des allocataires désireux de retrouver un emploi s'y rendaient : sur l'année 2004, plus de cent cinquante allocataires ont fréquenté plus ou moins régulièrement le centre; j'ai eu l'occasion d'en observer un petit tiers, chaque semaine durant quatre mois.

Une fois par semaine se tenait une réunion de synthèse destinée à aborder des sujets aussi divers que les objectifs fixés et atteints en termes de réinsertion dans l'emploi, des questions matérielles (laisser ou couper la musique visant à protéger la confidentialité des entretiens dans un lieu d'accueil en open space), le lancement de campagnes publicitaires pour faire connaître l'action du centre et y attirer les allocataires (sur la radio locale, lors de kermesses, au cours de visites dans les centres médico-sociaux), ou encore la discussion autour d'un cas particulier, par exemple un bénéficiaire considéré comme "problématique " pour les conseillers et la psychologue.

La recherche a consisté en une observation directe - parfois participante -, en l'enregistrement d'entretiens de recherche d'emploi et de bilans de compétence, lors de rendez-vous entre les conseillers emploi et les bénéficiaires, ainsi qu'en la réalisation d'entretiens semi-directifs avec ces derniers. Les documents internes du centre ont été mis à ma disposition, notamment les dossiers des bénéficiaires (appelés «clients») qui fréquentaient le centre : curriculum vitae, compte rendu d'entretiens avec la psychologue, suivi de la recherche d'emploi. J'ai également été conviée aux réunions d'équipe hebdomadaires; je pouvais assister aux entretiens avec les demandeurs d'emploi et rencontrer le personnel du centre.

Cette recherche n'a de prétention ni à la représentativité, ni à l'exhaustivité. Elle avait pour ambition de mettre au jour des processus d'assignation et de (re-) négociation identitaires. Au total, j'ai recueilli douze entretiens de vie enregistrés (auprès de neuf hommes et de trois femmes) et douze non enregistrés; j'ai observé une cinquantaine de "clients " en entretien de durée variable pouvant aller d'un quart d'heure à une heure avec leur conseiller ou avec la psychologue. Les dossiers du centre représentent une centaine de documents d'archives. Pendant plusieurs jours, parfois des semaines entières, je suis restée au centre alors qu'il connaissait un très fort ralentissement d'activité, faute de fréquentation. Ces moments étaient l'occasion de discuter avec les conseillers et de comprendre leur perception sociale sur la «clientèle» du centre et sur leurs doutes quant à leur mission sociale.

\section{Des hommes malades ou des chômeurs?}

Comme le rappelle Didier FASSIN (2000), à l'instar de toute situation sociale, les situations d'assistance participent à la construction identitaire (Messu, 1991). Observé dans une perspective goffmanienne, le face-à-face répété des allocataires et de leur conseiller constitue un moment d'interaction fort où la présentation de soi, face à des injonctions institutionnelles relayées par les travailleurs sociaux, est une exposition et une construction du soi, à la fois morale et sociale. Le passage du statut principal de handicapé à celui de demandeur d'emploi représente parfois un grand écart identitaire. À quelles conditions cette (ré-)assignation identitaire est-elle possible? Enfin, dans quelle mesure le genre, l'âge et, même si l'enquête n'a pu le faire apparaître ${ }^{(12)}$, l'appartenance ethnique jouent-ils en faveur ou à l'encontre de cette ré-assignation?

\section{Les effets des catégorisations administratives}

Le programme New Deal d'incitation à l'emploi comporte des effets administratifs et sociaux pervers, à la fois pour les conseillers emploi et pour les bénéficiaires de l'Incapacity Benefit. Ces derniers, catégorisés administrativement «adultes handicapés» avant 2001, tombent désormais sous le coup d'une double catégorie : le handicap et le chômage. Selon le dispositif mis en place par le gouvernement, la catégorie de demandeur d'emploi tend à prendre le devant de la scène identitaire. Même si, pour certains, l'inactivité sociale liée au handicap avait de facto valeur de chômage et qu'ils cherchaient du travail, la force de l'injonction officielle à retravailler, ne serait-ce qu'un peu, n'était pas la même, la gestion identitaire non plus. Confrontés au dispositif New Deal, ils sont enjoints de renégocier leur statut et la perception de leur handicap. S'ils sont là, c'est qu'ils veulent retravailler, qu'ils sont des assistés méritants. Mais s'ils retravaillent, dans quelle mesure continueront-ils d'être juridiquement et économiquement reconnus comme handicapés? Plus concrètement, ils s'exposent à perdre leurs allocations. L'autre problème concerne le simple fait de retravailler alors que la santé physique et/ou mentale est menacée. Ils se heurtent à l'injonction de devoir retravailler, mais «pas trop». Dans tous les cas, avant de retrouver un emploi, un travail sur soi - au sens goffmanien - est requis pour envisager ce changement de statut.

Le dispositif New Deal est conçu pour que les conseillers soient parties prenantes dans la reconversion mentale et sociale demandée aux allocataires ${ }^{(13)}$. Comme l'a montré Nicolas Duvoux (2009), une telle injonction à l'autonomie des assistés ${ }^{(14)}$ invite

(12) Alors qu'un tiers environ des allocataires sont d'origine indo-pakistanaise, en cinq mois d'observation, je n'ai rencontré qu'une femme rentrant dans cette catégorie.

(13) Sur le modèle néolibéral qui prévaut au Royaume-Uni, les centres $J O B S$, spécialement conçus pour les accueillir, ont un statut très particulier. Ils émanent du ministère du Travail, ont un financement conjoint avec les centres d'emploi classiques, dont leur personnel est le plus souvent issu. Les centres fonctionnent comme des PME avec des objectifs de résultats décidés par le ministère du Travail. Un objectif annuel de reclassement des allocataires est ainsi fixé non à l'échelle du centre, mais par conseiller.

(14) Le terme est à prendre dans l'acception qu'en donne Simmel (1988 [1907]) à propos des relations contractuelles régulières avec les services sociaux (FASSIN, 2000, p. 955; Duvoux, 2009, p. 17). 
à diversifier l'analyse sociologique des ancrages sociaux de l'identité. Cette dernière, dans cette interaction, se cristallise autour de trois processus : la négociation, la légitimité, et la confiance. Face à ce type d'injonction à l'autonomie, Didier Fassin (2000) souligne, quant à lui, la difficulté à évaluer la part de normalisation subie - et j'ajouterais d'intériorisation - et la part de détournement tactique des assistés dans leur reconstruction identitaire. Dans tous les cas, c'est bien la dynamique identitaire qui semble en question, dans ce jeu de négociation entre les individus catégorisés dans le groupe des assistés et l'institution de protection sociale et, plus concrètement, entre les bénéficiaires et les conseillers.

L'attitude des conseillers apparaît d'autant plus ambiguë qu'ils sont pris dans des contradictions : accompagner l'injonction de retour à l'emploi de leurs «clients», tout en tenant compte d'une part de leur handicap, d'autre part de leurs maigres ressources économiques. En outre, ils doivent exercer une contrainte en prônant le retour à l'emploi dans un contexte où l'injonction est prescriptive mais nullement répressive. Les bénéficiaires peuvent, s'ils le souhaitent, ignorer les incitations dont ils font l'objet sans que leur prestation sociale soit remise en cause, même si la plupart d'entre eux ont intérêt à trouver un emploi pour compléter leur revenu et sortir de la très grande pauvreté.

Pour observer l'amorce d'une négociation identitaire, voici l'extrait d'une observation d'entretien en mai 2004, entre une conseillère et un allocataire d'une cinquantaine d'années, revenu au centre pour la première fois après quatre ans d'absence.

«LA CONSEILlère : Que s'est-il passé? Cela fait longtemps...

L'AllocATAIRE : J'ai reçu une lettre du centre pour reprendre contact avec vous... J'ai de gros problèmes de genoux. Je me sens de reprendre à temps partiel, pas à temps plein.

LA CONSEILLÈRE : Qu'aimeriez-vous faire?

L'AlloCATAIRE : Cuistot... enfin, c'est le dernier boulot que $j$ 'ai fait en tout cas...

LA CONSEILlÈRE : Mais, plus précisément, qu'aimeriezvous faire?

L'Allocataire : Ben, j'peux pas savoir... ça fait tellement longtemps que je n'ai pas travaillé. C'est comme si je repartais à zéro.

(L'homme nous regarde en silence, comme s'il cherchait la réponse dans nos yeux.)

LA CONSEILLÈRE : Ce que vous aimeriez faire doit vraiment venir de vous. Et vous devez être réaliste. Venez consulter le classeur [d'offres] tous les jeudis. Asseyez-vous et lisez calmement toutes les offres. Un désir va émerger. Dans deux à trois semaines, ce sera plus clair. Nous avons aussi des offres sur l'ordinateur et vous prenez rendez-vous avec moi et vous vous demandez: "Est-ce que c'est ce que je voudrais faire?" Il y a aussi du travail bénévole. Vous seriez prêt à faire du travail bénévole?

(L'allocataire ne répond pas.)

LA CONSEILlÈRE: Vous avez un handicap pulmonaire, je vois. Bon, qu'aimeriez-vous faire dans l'idéal? Vous avez le temps. Si c'est trop dur, ne venez pas tous les quinze jours. Le premier objectif est de vous demander : "Qu'est-ce que je veux faire?" Déterminez ce que vous voulez faire. Qu'est-ce que vous n'aimez pas? Qu'est-ce qui vous intéresse?

L'Allocataire : Je ne veux pas travailler à temps plein. Je touche $316 £$ par mois.

(Nous regardons tous les trois les offres disponibles sur l'écran.)

La Conseillère : [...] Assistant de vente, agent d'entretien, travail administratif, vente, ménage. Bon, c'est vraiment juste pour vous donner une idée.

L'allocataire : Mais je ne peux ni me baisser ni m'accroupir!

LA CONSEILlÈre : Bon, vous devez vraiment vous demander ce que vous voulez faire, ce que vous pouvez et ne pouvez pas faire. »

La personne est ensuite invitée à prendre un rendez-vous avec la psychologue du centre pour un bilan de compétence. La conseillère prend l'agenda pour lui fixer un second rendez-vous avec elle, mais l'homme reste flou et dit qu'il repassera. Les modalités du premier entretien sont standardisées : placer le bénéficiaire face à la réalité, l'encourager à revenir au centre pour assurer le suivi de son dossier, sans pour autant lui mettre une pression trop forte. Il ressort de l'échange une contradiction qui, peu à peu, se fait jour et tourne au dialogue de sourds. Les offres d'emploi consultées censées «donner des idées» semblent inadaptées au handicap de la personne. Que vaut, dans ces conditions, l'injonction à prendre son destin en main en se demandant ce qu'on souhaiterait faire? La conseillère, en invitant l'allocataire à penser à un travail «dans l'idéal» tout en étant «réaliste» édicte d'ailleurs l'injonction paradoxale propre au dispositif(15) qui débouche souvent sur des emplois médiocres (LiNDSAY, McQuaid, 2004). Paradoxale, la démarche d'aide l'est à plus d'un titre, ce qui place les bénéficiaires face à une aporie identitaire. Dans ce travail de reconversion, le pouvoir de décision des allocataires s'avère très limité du fait de la double contrainte, économique et physique, qui pèse sur eux.

Dès le premier ou le second entretien de suivi, les conseillers calculent d'ailleurs, à l'aide d'un logiciel informatique, le nombre d'heures de travail maximal que la personne peut effectuer en augmentant ses revenus, sans pour autant amputer tout ou partie des différentes prestations sociales perçues.

(15) Nicolas Duvoux (2009) a observé ce même paradoxe à propos du contrat d'insertion. 


\section{Changer de statut : une aporie identitaire?}

Les allocations (handicap, logement et autres) ne suffisent pas pour vivre. En outre, l'enfermement dans la maladie contribue à renforcer le handicap, à le doubler parfois d'une dépression nerveuse. Il s'agit donc d'en sortir, moralement et économiquement.

L'argumentaire autour de la confiance en soi sature les discours de tous les acteurs de la négociation : la question est discutée à chaque réunion d'équipe; elle est constamment réaffirmée par les conseillers lors des entretiens avec les allocataires; enfin, ces derniers en parlent spontanément et très fréquemment en entretien, comme du premier grand obstacle à surmonter. Comme le remarque Lucie Davorne (2005) à propos de la mise en place du New Deal for Young People, le programme est considéré comme un succès dès lors qu'il permet une participation accrue à la vie sociale et une plus grande confiance en soi, même si l'objectif de retour à l'emploi n'est pas immédiatement atteint. Cela explique que ce thème soit omniprésent dans le discours des conseillers et des bénéficiaires : confiance en soi pour sortir du stigmate du «mauvais inactif», incrusté dans son handicap; confiance en soi pour convertir le stigmate de la maladie, qui rend inemployable, en chance d'emploi. De la confiance en soi à la confiance dans le système de reconversion et dans son mandataire, le conseiller emploi, le pas n'est pas pour autant aisément franchissable. Ce manque de confiance donne lieu à des séquences dont le décalage avec la réalité pourrait paraître burlesque, s'il n'était l'expression d'une forme d'aporie identitaire - l'impossibilité à s'identifier au statut de demandeur d'emploi - dans laquelle se trouvent bon nombre de bénéficiaires.

Par exemple, un allocataire de trente ans, qui fréquente le centre depuis plusieurs mois et y a même travaillé comme bénévole, est censé savoir que le $J O B S$ est une structure publique. Encouragé par sa conseillère lors d'une session de groupe, il téléphone plein d'allant pour un emploi et raccroche paniqué, sans savoir répondre quand l'employeur lui demande s'il téléphone de la part d'une agence de recrutement (à statut privé). La conseillère rappelle l'employeur pour expliquer la situation. Lors d'un second coup de téléphone, l'allocataire donne cette fois tous les renseignements requis et, après avoir raccroché, nous regarde effaré en nous disant qu'ils ne parviendront pas à le joindre, car son numéro de téléphone portable n'est plus valable depuis qu'il a perdu son téléphone dix jours auparavant.

La prise de confiance est d'autant plus difficile que le handicap prend, dans le discours, l'allure d'un stigmate. À plusieurs reprises, la question de sa dissimulation est soulevée. Les conseillers recommandent aux allocataires de ne pas le mentionner dès la première prise de contact. Eux-mêmes, lorsqu'ils téléphonent pour le bénéficiaire, profitent de ce que leur centre dépend des Jobcentre Plus pour expliquer qu'ils ont trouvé l'annonce sur le site de l'agence nationale pour l'emploi et qu'ils ont un candidat. De leur côté, les allocataires ont une relation ambiguë à l'égard de leur maladie, qu'ils perçoivent comme un obstacle à la réinsertion, comme l'exprime Mike.

"Je ne vais pas dire aux gens que j'ai des difficultés. [...] Si vous dites aux gens que vous êtes sous antidépresseurs, les gens prennent peur... Ils se disent que vous êtes un psychopathe quand vous leur dites que vous avez un problème psychologique. Alors les employeurs... [c'est encore pire].

- Aviez-vous peur de ne pas retrouver un travail?

- Bien sûr. Parce qu'on perd toute sa confiance en soi. Rien que le fait de sortir de la maison et de parler, ça me terrorisait. »

(Mike, trente ans, dépression chronique.)

Plusieurs prestataires vivent leur statut de manière contradictoire : comme un refuge, mais également comme un piège, à l'image de Jason.

«Comment vous définiriez-vous aujourd'hui? Vous voyez-vous avant tout comme chomeur ou avant tout comme souffrant d'un handicap?

- Vraiment les deux. Mais, peut-être avant tout comme sans-emploi parce que ça, c'est indiscutable. Pour ma maladie, ça a été plus compliqué. Ma maladie est un vrai problème parce que, alors que je croyais que ça allait s'améliorer petit à petit, ça ne s'est pas passé comme ça. C'est resté stationnaire... Je me disais :

"En arrêtant de travailler, ça va aller mieux, j'aurai moins de stress, ça va être plus facile." Mais, c'est pas vrai. Et certains jours, c'est même pire... Le pire, c'est de n'avoir pas de travail dans tout ça. [...] Et c'est un cercle vicieux...»

(Jason, cinquante-trois ans, technicien en chimie industrielle, grande fatigue chronique.)

Le passage du statut principal d'adulte handicapé à celui de demandeur d'emploi, puis de salarié dépend de plusieurs facteurs. Étudier les interactions entre des agents administratifs et leur public suppose la prise en compte des conditions et positions sociales de chacun-e des interactant-e-s (SiвLOT, 2006) ${ }^{(16)}$. En effet, le genre, la génération et le parcours des conseillers induisent un plus ou moins fort degré de proximité ou d'identification avec certains usagers. Autrement dit, l'assignation identitaire est fonction de l'interaction qui se noue et de la plus ou moins bonne relation avec les travailleurs sociaux (voir encadré 2). Elle dépend, en outre, de la situation de l'allocataire qui relève toujours de plusieurs catégories administratives et sociales - le handicap, le chômage ou l'inactivité,

(16) Même si, au fil des semaines, j'ai pu obtenir des éléments sur la trajectoire de certains conseillers, d'autres se sont montrés extrêmement réticents à parler d'eux. 
la catégorie socioprofessionnelle d'origine avec lesquelles, d'ailleurs, les conseillers emploi entendent composer.

Encadré 2
Une consultante qui valorise l'adaptation
à la flexibilité du marché du travail
Après un bilan de compétence auquel j'assiste,
Vera, trente-huit ans, conseillère emploi depuis
quatre ans, évoque avec moi le parcours de Jason,
cinquante-trois ans, qui n'a pas abordé avec elle la
question de son handicap mais décline les offres
qu'elle lui propose. Selon elle, le fait d'avoir fait
"le même travail pendant vingt ans, dans la même
entreprise est preuve d'une stagnation. Cela n'est
pas un plus dans son CV ". La conseillère en ques-
tion est une ancienne allocataire qui a fréquenté
le centre quelques années auparavant en tant que
demandeuse d'emploi. Avant un accident, elle a
connu de multiples emplois dont manipulatrice
radio et institutrice dans le secteur privé. Sa réin-
sertion réussie est comme le témoignage que sa
mission n'est pas vaine, sa présence au centre, de
l'autre côté de la barrière de l'emploi, la promesse
que l'Incapacity Benefit n'est pas une fatalité. C'est
à l'aune de sa propre trajectoire, faite de ruptures,
de reconversions multiples et de reconstruction,
que Vera catégorise l'allocataire. Dans ce qui m'ap-
paraît comme une volonté d'oblitérer le handicap et
le passé professionnel de Jason, afin qu'il puisse
"aller de l'avant", elle ne pose aucune question
concernant son handicap, sans comprendre pour-
quoi les offres qu'elle lui propose se heurtent à
un refus, ultime résistance au déclassement que
supposerait sa candidature.

Changer de statut requiert la conversion d'un regard, par la traversée d'un miroir social permettant la sortie de la carrière d'assisté et l'accession à une (nouvelle) carrière professionnelle normalisée. Étant donné qu'ils occupent, dans leur majorité, des emplois peu qualifiés, faiblement rémunérés ou bénévoles, cette conversion est coûteuse, voire périlleuse, financièrement et/ou psychologiquement. Si l'on assimile leur parcours identitaire à une carrière au sens beckérien du terme (BECKER, 1985), cette dernière se fait en au moins trois étapes : du statut d' "handicapé», ils accèdent au statut de "demandeur d'emploi» (17), étape à double statut nécessaire dans le dispositif les incitant à revenir un jour au statut d'actif occupé «en bonne santé».

Même si le dispositif institutionnel couvre le suivi de l'intégration professionnelle sur une

(17) Parfois, c'est en sens inverse, le chômage les conduit à demander et obtenir le statut d'adulte handicapé. durée de vingt-six semaines ${ }^{(18)}$, la reconversion dans l'emploi s'avère souvent non durable, pour ne pas dire impossible. L'analyse des moments de recherche d'emploi, des stages emploi, des entretiens montre qu'il s'agit d'une assignation identitaire institutionnalisée d'autant plus fragile que les garanties d'emploi pérenne à temps complet sont très faibles. En outre, l'institution entretient une ambiguïté puisque, officiellement, les adultes handicapés ne sont ni nommés ni comptabilisés comme «demandeurs d'emploi», alors qu'ils le deviennent de facto en fréquentant le centre emploi.

Ceux qui (s')en sortent le mieux sont les femmes, les moins âgés et les moins isolés socialement, selon les conseillers du JOBS, car ils réintègrent assez rapidement le marché du travail. Les conseillers les repèrent à leur motivation, à la somme de discussions informelles qui ont lieu lors de la fréquentation au centre, et à la régularité de la recherche. A contrario, beaucoup d'allocataires disparaissent brutalement, pendant plusieurs semaines ou plusieurs mois, quand ce n'est pas définitivement. Pour certains conseillers emploi, la présence régulière d'un membre de la famille (un conjoint, un oncle, un frère, une tante, etc.) accompagnant le candidat à l'emploi (19) est une garantie supplémentaire de sa détermination. Même dans les cas de réussite à long terme, les personnes ainsi réinsérées s'intègrent au prix du renoncement à leur identité de malade qui, pour inconfortable et stigmatisée qu'elle soit, constitue souvent un garde-fou au regard d'une plus grande précarité sur un marché du travail incertain et qui, paradoxalement, peut les rassurer. Plus qu'un grand écart, la tension entre les deux identités se résout par un saut identitaire sans garantie que l'identité d'actif occupé soit finalement plus valorisante et rémunératrice que celle d'inactif handicapé.

\section{La part du genre et de l'âge des allocataires}

«L'épreuve du chômage» (SCHNAPPER, 1994) est vécue sur un mode différent en fonction du sexe, de l'âge, de l'origine ethnique et géographique, de la catégorie sociale, du handicap, et de la durée du chômage. De manière générale, l'Incapacity Benefit touche une majorité d'individus aux deux extrémités de la pyramide des âges, en affectant les jeunes et les plus âgés parmi ceux en âge de travailler,

(18) Un conseiller à temps complet a la charge d'enregistrer le suivi de cent quarante «clients». Parmi ces derniers, quarante personnes, auxquelles le centre garantit un suivi de six mois, doivent avoir trouvé un emploi.

(19) Aucune des femmes observées au centre n'est venue accompagnée. En revanche, la moitié des hommes environ venaient accompagnés de leur compagne ou d'un parent. Les hommes les mieux dotés en capital scolaire et culturel venaient également seuls. 
autrement dit les catégories d'âge les plus vulnérables sur le marché du travail.

Parmi les allocataires qui fréquentent ou ont fréquenté le lieu de l'enquête, j'ai rencontré une majorité d'hommes ouvriers d'origine anglosaxonne, même si j'ai aussi croisé des femmes et quelques rares hommes diplômés. La moindre présence des femmes au centre s'explique par leur faible part parmi les bénéficiaires de l'Incapacity Benefit jusqu'à une date récente. À cet égard, l'enquête donnerait des résultats différents aujourd'hui, étant donné l'accroissement de leur inscription dans l'invalidité en l'espace de six ans, presque à égalité avec les hommes à la fin des années 2000 (BEATTY et al., 2009).

Concernant la quasi-absence d'hommes d'origine indo-pakistanaise, inversement proportionnelle à leur part importante parmi les allocataires de l'Incapacity Benefit dans la région étudiée, deux hypothèses peuvent être formulées : d'une part, elle serait due au principe de volontariat du programme New Deal for Disabled People qui suppose une familiarité avec l'institution et les agents de l'État, rendue plus complexe pour les minorités ethniques issues de l'histoire coloniale britannique. D'autre part, elle s'expliquerait par une plus grande participation des bénéficiaires indo-pakistanais à l'économie informelle. Leur activité «au noir» serait ainsi à l'origine de leur réticence à s'inscrire sur un marché du travail précaire et mal payé menaçant leur allocation maladie. Dans les deux cas, la faible fréquentation des allocataires d'origine indo-pakistanaise serait motivée par un contournement du contrôle indirect que représenterait l'inscription au JOBS par rapport au travail et à la maladie.

En ce qui concerne le genre, une majorité d'hommes est concernée par l'assistance sociale et par le programme de réinscription dans l'emploi. La privation d'activité, redoublée par la stigmatisation du handicap dont ils souffrent, les place dans une position de vulnérabilité différente des femmes dans des situations comparables. En effet, leur virilité est doublement atteinte : par la privation d'emploi et par la déviance que représente leur maladie avérée, et dont les troubles sont souvent accompagnés de syndromes psychologiques (grande fatigue mentale, dépression voire troubles psychiques graves). $\mathrm{Si}$, pour les femmes, ces symptômes sont socialement perçus comme «plus acceptables», ils sont vécus comme honteux, dégradants et «dévirilisants» par les hommes. Ce phénomène a été étudié en France sous l'angle de l'identité de genre, en particulier chez les ouvriers (Schwartz, 1990) et chez les cadres autodidactes (Pochic, 2000). Comme dans le bassin sidérurgique du nord de la France, cette «dévirilisation» des anciens ouvriers apparaît d'autant plus forte dans le nord de l'Angleterre que la construction du masculin et du féminin a la réputation d'y être très stéréotypée et que l'identification au masculin, traditionnellement liée à un travail rude et physique (dans les mines et les industries), y a été historiquement structurante jusqu' aux années Thatcher ${ }^{(20)}$. Dans l'imaginaire social, les hommes du nord sont peu loquaces, caustiques, ils ont la réputation d'ironiser avec humour sur la dureté de leurs conditions de vie, notamment dans des lieux collectifs de socialisation comme le pub, n'ont pas l'habitude de se plaindre, et «ne tombent pas malades», sous peine de passer pour des «mauviettes» ou des «femmes»... Il s'agit d'une construction du masculin, sur le modèle du travailleur industriel, confronté à une pénibilité du travail qui sert aussi de mode de valorisation (Pezé, 2002).

L'âge - à la fois indicateur d'une position dans le cycle de vie et élément de représentation de soi - joue comme un facteur supplémentaire dans la vulnérabilité de ces individus(21). Des sociologues britanniques indiquent que $65 \%$ des hommes de plus de cinquante ans touchent l'Incapacity Benefit et que $48 \%$ des bénéficiaires de l'Incapacity Benefit ont plus de cinquante ans (RIACH, LORETTO, 2009, p. 104). Pour ces derniers, les handicaps chômage et maladie - s'additionnent et ce cumul conduit à renforcer ce que GOFFMAN appelle une «identité blessée» (voir encadré 3). Le discours des conseillers et des allocataires coïncide pour dire à la fois l'inemployabilité des jeunes, plus inexpérimentés, et la difficulté à rebondir quand on est un homme de plus de cinquante ans. Cela place tous les allocataires en demeure de devoir accepter des salaires bien inférieurs à ce qu'ils touchaient avant de basculer dans l'invalidité pour ceux qui ont travaillé, et des emplois parfois sans rapport avec ce qu'ils faisaient avant. Ces changements de statut conduisent à transformer le regard social, à tel point que certains jouent de l'ambiguïté rendue possible par leur âge pour ne pas annoncer au voisinage qu'ils sont en inactivité et faire croire qu'ils sont à la retraite.

(20) Pour une transcription littéraire de cette opposition entre les hommes du nord et ceux du sud, voir le roman d'Elizabeth Gaskell (1854), North and South, Londres, Penguin, ou, plus récemment, les travaux de l'anthropologue Kate Fox (2005), Watching the English. The Hidden Rules of English Behaviour, Londres, Hodder and Stoughton LTD.

(21) Pascale Jamoulle (2005) a par exemple étudié la négociation d'identité virile de jeunes précaires et montré combien l'âge et l'entrée dans la vie adulte étaient structurants de conduites décalées ou à risque. 


\section{Encadré 3}

\section{Entre inactivité honteuse et déclassement imposé : le tiraillement identitaire}

Le cas de Jason est exemplaire à ce titre : à cinquante-trois ans, cet homme, ancien technicien, a vu un médecin après avoir éprouvé les premiers symptômes de grande fatigue liés à un gros stress au travail. Celui-ci faisait suite à une fraude industrielle de son supérieur à propos d'une technique chimique que Jason avait lui-même mise au point. À la suite de cela et d'une erreur de diagnostic du médecin du travail, il a été licencié avec une indemnité conséquente. Bien que confus concernant l'historique de sa maladie - il est depuis dix-huit mois en inactivité pour invalidité -, il ne cesse de parler de son âge, de ce que cela signifie par rapport aux attentes et besoins des entreprises, au regard social, à sa propre perception des choses. II est conscient des conséquences de sa réinsertion sur le marché du travail : déqualification professionnelle, déclassement salarial sont inévitables et, au moment de l'entretien, il ne peut accepter cette réalité tout en étant préoccupé par sa «mauvaise employabilité " à plus de cinquante ans. De ce fait, il ne parvient pas à se projeter dans l'avenir, encore moins à redéfinir un projet professionnel. Symétriquement, il est imprécis dans ses indications de dates (dates exactes des différentes étapes dans l'obtention de l'allocation, de sa première venue au centre) comme si ce qu'il vivait comme un échec passé hypothéquait l'avenir. Plus diplômé que les autres «clients " du centre emploi, il apparaît décalé lors des sessions de formation en groupes (technique de recherche d'emploi, rédaction de $\mathrm{CV}$, valorisation des compétences) où il se montre néanmoins l'un des plus assidus. De même, il dit se sentir en porte-à-faux face à la conseillère qui lui propose les mêmes postes que ceux qu'elle offre à d'autres hommes en fonction de leur âge et de leur pathologie. Elle-même est aux prises avec une contradiction : Jason est l'un des plus sérieux et des plus motivés parmi ceux qui fréquentent le centre mais elle le perçoit comme inadapté par rapport aux dispositifs de réinsertion d'une part, et par rapport aux postes disponibles qu'elle lui propose d'autre part.

Aux yeux de Jason, âge, handicap et durée du chômage se cumulent et menacent ses chances de retrouver un emploi digne de ses compétences. Il est tiraillé entre son identité professionnelle passée, dont il n'a pas fait totalement le deuil, et son statut d'inactif, chroniquement fatigué et très déprimé au moment de l'entretien. Comme l'a remarqué Olivier SCHWARTz (1990) à propos de la vie privée des ouvriers du nord, il existe des «lieux de recomposition masculine», sortes d'entre-deux entre le lieu de travail et la famille, comme la pêche, le jardinage, le bricolage. Ces lieux, qui assurent une fonction de soupape de l'identité masculine, se trouvent fragilisés en cas de perte d'emploi. Comme le note SCHWARTZ (1990, p. 358), «un homme au chômage est coupé des lieux où se conquiert et se réaffirmait jusque-là sa légitimité», y compris hors travail. Au cours de l'entretien, les activités de jardinage et de bricolage, autrefois plaisirs de loisir, apparaissent désormais plus ambiguës voire illégitimes à Jason en raison d'un rapport au temps qui semble à la fois suspendu et distendu. Le temps vide et l'espace fermé décrits par SCHWARTZ se retrouvent ici comme des motifs de l'illégitimité masculine liée à l'absence d'emploi et à l'ennui. Jason insiste d'ailleurs, comme quelques autres, sur le fait qu'il s'attache à garder un emploi du temps en mettant son réveil à la même heure que s'il partait au travail, tandis qu'il reconnaît se cacher des voisins pour éviter les questions indiscrètes et les commentaires, et donc s'adonner avec retenue et discrétion au jardinage et au bricolage.

Le facteur de l'âge n'explique pas tout. Il mériterait d'être corrélé à l'occupation antérieure et au niveau de diplôme et de qualification, à l'expérience d'emploi. La perte des repères et la rupture identitaire semblent d'autant plus coûteuses que l'emploi précédent était stable, bien rémunéré, voire socialement valorisant comme l'a montré Sophie Pochic (2000) sur des cadres promus licenciés en fin de carrière. John, ouvrier de formation de cinquantetrois ans lui aussi, a occupé plusieurs emplois après son licenciement à l'usine : conducteur de poids lourds, cariste, gardien dans une riche propriété. Durant la période où il fréquenta le centre, comparé à Jason qu'il rencontre lors d'une demi-journée de formation à la recherche d'emploi, il affiche une attitude plus détachée, voire «inconséquente» selon sa conseillère qui le soupçonne d'ailleurs de travailler de manière informelle comme jardinier et homme à tout faire.

En regard de Jason et John, Mike fait partie des «jeunes» allocataires du centre (voir encadré 4). Son rapport à l'inactivité et au handicap s'explique à la fois par son âge, son expérience antérieure dans des emplois alimentaires, et la nature de son handicap.

À la différence de Jason, le rapport qu'entretient Mike avec l'emploi semble avant tout conditionné par son handicap, la dépression, et non par son passé ou son projet professionnels. Les ruptures et reconstructions identitaires articulent différentes logiques liées au genre, à l'âge, au handicap mais aussi à la catégorie socioprofessionnelle et à l'emploi antérieur, comme le montre la trajectoire de Steve (voir encadré 5). 


\section{Encadré 4}

\section{Le retour au travail comme facteur de stabilisation sociale}

Mike a trente ans et est divorcé, père d'une fillette de sept ans. Le jour de l'entretien, il vient d'apprendre qu'il a été retenu pour un contrat de douze mois à temps partiel, dix heures par semaine. Cet emploi lui permet de conserver ses allocations. La veille, il a appris qu'il avait également obtenu un logement alors qu'il avait dû retourner habiter chez ses parents à la suite de son divorce. Mike est atteint de dépression depuis quatre ans mais évoque des problèmes psychologiques qui remonteraient à l'enfance. Contrairement à Jason et à des allocataires plus âgés, il semble avant tout s'identifier à son handicap qui prend une grande place dans l'entretien. Son attitude d'identification secondaire à son statut de chômeur s'explique peut-être par ses faibles qualifications professionnelles, par un parcours de petits boulots fait de multiples ruptures de contrat, et par le fait qu'il vient de retrouver un emploi le jour où je le rencontre. II a quitté l'école à seize ans et a occupé toutes sortes d'emplois dont les plus longs ont été livreur, pendant trois ans, et cariste dans la grande distribution pendant huit ans, jusqu'en 1999, où il tombe en grave dépression. Sa dépression l'a écarté du marché du travail, même s'il a occupé des emplois bénévoles, notamment auprès de son beau-frère, pour tenter de reprendre confiance en lui. Il semble très fortement inséré dans le tissu familial qui le soutient dans sa recherche d'emploi. Son oncle l'accompagne régulièrement au centre. Sa sœur l'aide beaucoup moralement et financièrement, et sa fille qu'il voit régulièrement apparaît comme un soutien important. Hormis le facteur économique, le retour à l'emploi est pour lui le moyen de structurer un rythme quotidien qu'il a perdu avec la maladie, et non un mode de reconquête d'une identité positive par le travail qu'il aurait perdue même si, indéniablement, avoir un emploi contribuera à reconstruire une certaine confiance en lui.

\section{Encadré 5 \\ Des aspirations professionnelles parfois limitées par un handicap lourd}

Steve se situe, en termes de classe d'âge, entre Mike, Jason et John. II a quarante-quatre ans au moment de l'entretien et représente le candidat à l'emploi le plus diplômé du JOBS. Interrogé sur la manière dont il se définirait par rapport au travail, il répond qu'il se voit comme un «éternel étudiant». Détenteur d'une thèse $(P h D)$ en informatique obtenue en 2003 , après cinq ans, il est atteint de troubles psychologiques graves et a été diagnostiqué "schizophrène" par les services de santé publique. II reconnaît avoir choisi de reprendre des études avant tout par intérêt personnel et parce qu'il ne trouvait pas d'emploi adapté à sa santé mentale.

\begin{abstract}
Le statut d'allocataire de l'Incapacity Benefit semble avoir joué un rôle favorable dans l'attribution d'une allocation de thèse de $10000 £$ par an pendant les trois premières années. Ensuite, l'assurance maladie et les économies faites au cours de ces années d'études lui ont permis de tenir deux ans supplémentaires durant lesquels il a achevé son doctorat. Son parcours professionnel antérieur est chaotique; il le décrit comme «le mauvais emploi au mauvais moment "ainsi qu'«inadapté " à sa santé mentale. À l'époque, un premier diagnostic de TOC (troubles obsessionnels compulsifs) est posé. Quoi qu'il en soit, il n'a jamais gardé un emploi plus de quelques mois dans l'ingénierie mécanique, son domaine de formation initiale, et a connu une sévère période de dépression nerveuse durant près de cinq ans au milieu d'un parcours à bâtons rompus qu'il a du mal à reconstituer, alors qu'il se révèle extrêmement précis sur les dates et les faits relatifs à ses études. II est depuis sous forte médicamentation et rencontre des troubles de la concentration qui constituent, à ses yeux et de l'avis de son conseiller, un obstacle pour conserver un emploi à la hauteur de ses compétences. Face au système, Steve se sent "décalé", ce que confirme son conseiller emploi. En effet, il souhaiterait occuper un poste d'assistant de recherche à l'Institut de technologie de la ville, emploi qu'il a peu de chances d'obtenir compte tenu de son parcours et de son handicap. II envisage de ce fait de s'inscrire dans le programme NDDP en postulant pour un travail comme bénévole, qu'il n'est pas non plus certain d'obtenir au vu de ses difficultés d'insertion sociale et de concentration.
\end{abstract}

Au regard des allocataires visés par le dispositif New Deal for Disabled People, Steve présente un profil en décalage dans la mesure où il ne correspond pas aux catégories jugées «employables» par l'institution, soit les $60 \%$ de bénéficiaires jugés aptes à «retravailler sous certaines conditions» (BEATTY et al., 2009). Il appartient au contraire aux $10 \%$ atteints de handicap lourd. Sa présence au centre est le résultat d'une initiative personnelle. Contrairement à d'autres, il n'a pas reçu de lettre l'invitant à se présenter pour un bilan de compétence mais a rencontré des conseillers lors d'une journée portes ouvertes et s'est présenté spontanément au $J O B S$, porteur d'une attente en décalage avec ce que le centre peut offrir. Sa présence confirme la difficulté rencontrée lors de la mise en place d'un dispositif de workfare qui prétend individualiser les parcours de réinsertion alors que les postes offerts sont standardisés et tournés vers des emplois peu qualifiés, à temps partiel et sans réelle perspective d'avenir. 


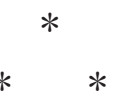

En conclusion, ces quelques portraits posent la question du pari que constitue la réinsertion professionnelle d'individus qui se vivent - parfois exclusivement - comme malades. Ils illustrent aussi le paradoxe à vouloir prétendre réinscrire dans des bad jobs (emplois pourris) ou des deadend jobs (emplois impasses) des personnes qui se sont «construit» un statut d'adulte handicapé pour échapper à cette instabilité du travail, ou, dans le cas de Jason, échapper «par la maladie» à la souffrance au travail et à un déclassement statutaire et salarial. L'ensemble de l'enquête montre enfin toute la difficulté à souscrire à une nouvelle assignation identitaire quand celle-ci paraît économiquement incertaine et socialement peu valorisée.

De manière plus large, l'enquête présentée ici renvoie aux contradictions du modèle libéral britannique. Si pendant deux décennies, le taux de chômage du Royaume-Uni a été l'un des plus faibles d'Europe, en revanche son taux d'inactivité est demeuré plus élevé que dans beaucoup de pays européens. Les programmes New Deals ne sont pas parvenus à juguler cette inactivité et les ouvriers masculins semblent avoir montré que tout était préférable à leur participation à la dérégulation du marché du travail, même la retraite anticipée de plusieurs années, même l'invalidité, peut-être plus acceptable à leurs yeux que le statut de chômeur... Cette inactivité dans la maladie s'est d'ailleurs, depuis la toute fin des années 2000, étendue aux femmes, phénomène inédit jusque-là. Correspondant à un processus différent du modèle masculin lié à la désindustrialisation, il s'expliquerait par une croissance de l'activité féminine et donc, paradoxalement, par une augmentation du nombre de femmes dans l'Incapacity Benefit pour échapper aux emplois précaires, manière de dire la résistance au modèle libéral anglo-saxon. 


\section{Bibliographie}

Alcock P., Beatty C., Fothergill S. et al. (2003), Work to Welfare. How men become detached from the labour market, Cambridge, Cambridge University Press.

Angeloff T. (1999), «Des miettes d'emploi : temps partiel et pauvreté», Travail, genre et sociétés, nº 1, pp. 43-70.

BARbier J.-C., Brygoo A. (2000), «Handicapés et emploi : une difficile comparaison des politiques en Europe», 4 Pages, no 40.

BARnAY T. (2008), «Chômage et invalidité après cinquante ans : deux dispositifs alternatifs de sortie de l'emploi pour les seniors en mauvaise santé?», Économie et Statistique, no 411, pp. 47-63.

Beatty C., Fothergill S., Houston D. et al. (2009), Women on Incapacity Benefit, rapport de recherche pour l'Economic and Social Research Council.

Becker H. (1985 [1963]), Outsiders, Paris, Métailié.

Bellaby, P. (1999), Sick from work: the body in employment, Aldershot, Ashgate.

Benarrosh Y. (2003), «Les trappes d'inactivité : chômage volontaire ou chômage de résistance?», Travail et Emploi, no 95, pp. 73-85.

Davorne L. (2005), «Le New Deal for Young People: des évaluations contrastées», Travail et Emploi, no 101, pp. 7-19.

Demazière D. (2003), Le chômage. Comment peut-on être chomeur?, Paris, Belin.

Demazière D. 1992), Le chômage en crise? La négociation des identités des chômeurs de longue durée, Lille, PUL.

Denman J., McDonald P. (1996), «Unemployment statistics from 1881 to the present day ", Labour Market Trends, Vol. 1, no 104, pp. 5-18.

Divay S., Perez C. (2010), «Conseiller les actifs en transition sur le marché du travail : la généralisation de pratiques professionnelles différenciées », SociologieS, p. 60-76.

Dubet F., Vérétout A. (2001), «Une "réduction" de la rationalité de l'acteur. Pourquoi sortir du RMI?», Revue française de sociologie, Vol. 42, no 3, pp. 407-436.

Duvoux N. (2009), L'autonomie des assistés. Sociologie des politiques d'insertion, Paris, PUF.

Duvoux N., Paugam S. (2008), La régulation des pauvres, Paris, PUF.

Ebersold, S. (2004), «L'insertion ou la délégitimation du chômeur», Actes de la recherche en sciences sociales, no 154, pp. 94-108.

Eсотес (2006), Incapacity Benefit Related Worklessness in the North of England: A Review of Current Evidence. A report to the North West Development Agency.
FAssin D. (2000), «La supplique. Stratégies rhétoriques et constructions identitaires dans les demandes d'aide d'urgence», Annales. Histoire, sciences sociales, Vol. 55, no 5, pp. 951-981.

Fox (2005), Watching the English. The Hidden Rules of English Behaviour, Londres, Hodder and Stoughton LTD.

Freyssinet J. (2004), Le chômage, Paris, La Découverte, coll. «Repères».

Gaskell E. (1854), North and South, Londres, Penguin.

Gollac M., Volkoff S. (2006), «La santé au travail et ses masques», Actes de la recherche en sciences sociales, no 163 , pp. 4-16.

Jamoulle P. (2005), Des hommes sur le fil. La construction de l'identité masculine en milieux précaires, Paris, La Découverte.

Kazimirski A., Pires C., Shaw A., Sainsbury R. and Meah A. (2005), «New Deal for Disabled People: Eligible Population Survey, Wave Three», DWP Research Report, no 324: http://research.dwp.gov.uk/asd/asd5/rports20052006/rrep324.pdf.

Khlat M., Sermet C. (2011), «Quels liens entre maladie et perte d'emploi?», Santé et Travail, no 73, pp. 1-2.

Lefresne F., BegG I. (2008), «Le marché du travail britannique : mythe et réalité du modèle néolibéral», Revue de l'Ires, $\mathrm{n}^{\circ}$ 58, pp. 111-143.

LEFRESNE F. (2008), «Les avatars d'une indemnisation du chômage faiblement protectrice : le cas britannique», Chronique Internationale de l'Ires, no 115, pp. 112-123.

LindSAY C., McQuaID R. (2004), «Avoiding the "McJobs". Unemployed job seekers and attitudes to service work», Work Employment and Society, Vol. 18, $\mathrm{n}^{\circ} 2$, pp. 297-319.

Messu M. (1991), Les assistés sociaux. Analyse identitaire d'un groupe social, Toulouse, Privat.

Office for National Statistics (1997), Labour Force Survey Historical Supplement, Londres, The Stationary Office.

Paugam S. (2007), Le salarié de la précarité. Les nouvelles formes de l'intégration professionnelle, Paris, PUF.

Paugam S. (1993), La société française et ses pauvres. L'expérience du revenu minimum d'insertion, Paris, PUF.

Pezé M. (2002), Le deuxième corps, Paris, La Dispute, coll. «Le genre du monde».

Pochic S. (2000), «Comment retrouver sa place? Chômage et vie familiale de cadres masculins », Travail, genre et sociétés, no 3, pp. 87-108. 
Porcellato L., Carmichael F. et al. (2010), «Giving older workers a voice: constraints on the employment of older people in the North West of England», Work Employment and Society, Vol. 24, no 1, pp. 85-103.

Riach K., Loretto W. (2009), «Identity work and the "unemployed" worker: age, disability and the lived experience of the older unemployed», Work Employment and Society, Vol. 23, no 1, pp. 102-119.

RoInsard N. (2007), La Réunion face au chômage de masse. Sociologie d'une société intégrée, Rennes, PUR.
SAlais R., BAVEREz N., (1986), L'invention du chômage : histoire et transformations d'une catégorie en France des années 1880 aux années 1980, Paris, PUF.

Schnapper D. (1994 [1981]), L'épreuve du chômage, Paris, Gallimard.

Siblot Y. (2006), Faire valoir ses droits au quotidien. Les services publics dans les quartiers populaires, Paris, Presses de Sciences Po.

Schwartz O. (1990), Le monde privé des ouvriers. Hommes et femmes du Nord, Paris, PUF. 